\title{
Herschel PACS and SPIRE observations of TW Hydrae association brown dwarf disks ${ }^{\star}$
}

\author{
B. Riaz ${ }^{1}$ and J. E. Gizis ${ }^{2}$ \\ ${ }^{1}$ Centre for Astrophysics Research, Science \& Technology Research Institute, University of Hertfordshire, Hatfield, AL10 9AB, UK \\ e-mail: b.riaz@herts.ac.uk \\ 2 Department of Physics and Astronomy, University of Delaware, Newark, DE 19716, USA
}

Received 2 May 2012 / Accepted 11 June 2012

\begin{abstract}
We present Herschel SPIRE observations of the TW Hydrae association (TWA) brown dwarf disks SSSPM J1102-3431 (SS1102) and 2MASSW J1207334-393254 (2M1207). Both disks are undetected in the SPIRE 200-500 $\mu \mathrm{m}$ bands. We analyzed the archival PACS data and found no detection of either source in the $160 \mu \mathrm{m}$ band. On the basis of radiative transfer modeling, we estimate an upper

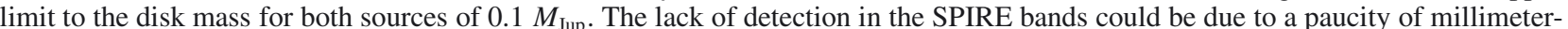
sized dust grains in the 2M1207 and SS1102 disks. We also report a non-detection for the brown dwarf 2MASS J1139511-315921 (2M1139) in the PACS 70 and $160 \mu \mathrm{m}$ bands. We argue for the presence of a warm debris disk around 2M1139, based on an excess emission observed at $24 \mu \mathrm{m}$. The mid-infrared colors for $2 \mathrm{M} 1139$ are similar to the transition disks in the Taurus and Ophuichus regions. A comparison of the brown dwarf disk masses over a 1-10 Myr age interval suggests that there is a decline in the disk mass with the age of the system.
\end{abstract}

Key words. accretion, accretion disks - brown dwarfs - circumstellar matter

\section{Introduction}

The TW Hydrae association (TWA) has been under observation since its identification by Kastner et al. (1997). This young $\left(10_{-2}^{+5}\right.$ Myr; Barrado y Navascués 2006) and nearby ( 53 pc, Gizis et al. 2007) association now has more than 30 confirmed members, in both stellar and sub-stellar regimes, with a few members displaying signatures of being in the T Tauri phase. Given its proximity and a critical intermediate age when most disks begin to show a transition to the debris phase, TWA is well-suited for the study of disk evolution and planet formation. Using ground-based mid-infrared (mid-IR) observations, Jayawardhana et al. (1999) and Weinberger et al. (2004) were able to detect excess thermal emission at 10, 12, and $18 \mu \mathrm{m}$ for 4 TWA members (TW Hya, Hen 3-600, HD 98800A, and HR 4796A). However, most of the other members do not show any evidence of circumstellar dust at these wavelengths, indicating that there has been a cleansing of dust in the inner disk regions on a timescale of $\leq 10$ Myr. A Spitzer survey by Low et al. (2005) using the sensitive MIPS instrument revealed the presence of cold debris disks around two TWA members, TWA 7 and 13 , at $70 \mu \mathrm{m}$, that had remained undetected by IRAS and other previous surveys. Among the brown dwarf members of TWA, excess emission has been detected at mid- and far-infrared (far-IR) wavelengths for 2MASSW J1207334-393254 (2M1207) and SSSPM J1102-3431 (SS1102), while a small $24 \mu \mathrm{m}$ excess has been reported for 2MASSW J1139511-315921 (2M1139), suggesting the presence of a transition disk around this source (Riaz et al. 2006; Riaz \& Gizis 2008). By transition disks, we imply a disk with an inner opacity

\footnotetext{
* Herschel is an ESA space observatory with science instruments provided by European-led Principal Investigator consortia and with important participation from NASA.
}

hole. The inner disk in such cases may consist of optically thin dust, or could be completely devoid of circumstellar material. Owing to a reduced opacity in the inner disk regions, transition disks exhibit photospheric emission shortward of a certain midIR wavelength, depending on the size of the inner disk hole. On the basis of the overall disk fractions and the strength in the midIR excess emission, Riaz \& Gizis (2008) suggested longer disk dissipation timescales for the brown dwarfs in TWA. This association has proven to be an important field to study the formation and evolutionary differences, if any, in stellar and sub-stellar objects, and their disk evolution timescales.

We present ESA Herschel Space Observatory (Pilbratt et al. 2010) SPIRE (Griffin et al. 2010) observations at 200-500 $\mu \mathrm{m}$ for SS1102 and 2M1207, and PACS (Poglitsch et al. 2010) 70 and $160 \mu \mathrm{m}$ observations for $2 \mathrm{M} 1139$. Observations at far-IR wavelengths for SS1102 and 2M1207 can help us to constrain the model fits and obtain more robust estimates of the disk mass and outer disk radius, while PACS observations of 2M1139 are important for probing the presence of cold dust material in this transition disk. Our Herschel observations are presented in Sect. 2. Results of the radiative transfer modeling are presented in Sect. 3. Section 4 provides a comparison of the TWA brown dwarf disk masses with the higher mass stars in this association and with the younger brown dwarf disks in Taurus, along with a discussion of the nature of the transition disk around 2M1139.

\section{Observations and data analysis}

We obtained SPIRE photometric observations for SS1102 and $2 \mathrm{M} 1207$ in the 250, 350, and $500 \mu \mathrm{m}$ bands, using the smallmap mode (ObsID: 1342234825/1342223257). The small-map mode covers a $5^{\prime}$ diameter circle, with a fixed scan angle of $42.4^{\circ}$ and a fixed scan speed of $30^{\prime \prime} \mathrm{s}^{-1}$. We requested a setup of 
25 repetitions, resulting in a total on-source integration time of $925 \mathrm{~s}$. For 2M1139, PACS photometric observations were obtained in the scan-map mode in the blue $(70 \mu \mathrm{m})$ and the red $(160 \mu \mathrm{m})$ filters. Two different scan-map angles of $45^{\circ}$ and $63^{\circ}$ were used (ObsID: 1342224188/89). Both scans were obtained at a medium scan speed of $20^{\prime \prime} / \mathrm{s}$, with a cross scan step of $5^{\prime \prime}$ and scan leg length of $3^{\prime}$. The number of scan legs was 8 for the $63^{\circ}$ scan, and 4 for the $45^{\circ}$ scan.

Data analysis for all sources was performed using the rectangular-sky photometry task provided in the Herschel Interactive Processing Environment (HIPE version 8.0.1). We analyzed the final pipeline-processed data (the "Level 2" products). The rectangular photometry task applies aperture photometry to both a circular target aperture and a rectangular sky aperture. For 2M1139, photometry was performed on the mosaic of the two scan maps. The sky intensity was estimated using the median-sky estimation algorithm. Since the sky intensity could vary with location, we selected 4 different background regions around the target (each of $30 \times 30$ pixel area) and measured the sky-subtracted source flux in each sky region. The final source flux and error is the mean and the standard deviation of these 4 flux measurements. The flux errors are consistent with the values obtained at the source location from the "error" image provided in the Level 2 products.

\section{1. $S S 1102$}

SS1102 is undetected in all SPIRE bands (Fig. 1). The positional accuracy as determined from the actual RA, Dec and the RANominal, DecNominal in the metadata is $\sim 1$ pixel (or $\sim 6^{\prime \prime}$ in the $250 \mu \mathrm{m}$ band). We calculated the upper limits using a pixel radius of 2 pixels centered on the nominal position of SS 1102. Harvey et al. (2012) reported a detection for SS1102 in the PACS $160 \mu \mathrm{m}$ band, at a $\sim 7 \mathrm{mJy}$ level. We analyzed their PACS data, and found no source detection at either the nominal or actual position of the target (Fig. 2). The flux level at the target location is $\sim 0.1-0.2 \mathrm{mJy}$, and is the same as found in some of the confusion noise dominated regions seen in the image. The offset between the actual and nominal positions in the metadata is $\sim 1.4^{\prime \prime}$, or 3.5 pixels. However, there is no source detection at the $2-\sigma$ level even when the offset is taken into account. The $160 \mu \mathrm{m}$ flux measurement for SS1102 reported by Harvey et al. (2012) is thus incorrect. Our analysis of SS1102 in the PACS $70 \mu \mathrm{m}$ band, however, is consistent with the measurement reported by Harvey et al. (2012). Table 1 lists the updated fluxes for SS1102.

\section{2. $2 M 1207$}

In Riaz et al. (2012a), we reported a detection for 2M1207 in the SPIRE bands of 250 and $350 \mu \mathrm{m}$, but a comparison now with the PACS images indicates that this was a misidentification. The revised SPIRE fluxes (upper limits) for 2M1207 were presented in Riaz et al. (2012a), where we noted that the source may be unresolved in the SPIRE bands, given the large positional offset of $\sim 14^{\prime \prime}$. However, the contamination from the nearby bright object would likely dominate the composite photometry in such a scenario. The SPIRE upper limits were determined by measuring the emission at the nominal position for 2M1207, without considering the positional offset.

We also analyzed the PACS 70 and $160 \mu \mathrm{m}$ observations of 2M1207. Our $70 \mu \mathrm{m}$ measurement is consistent with the flux reported by Harvey et al. (2012). In the $160 \mu \mathrm{m}$ image, there is some diffuse emission seen at a distance of 2 pixels $\left(\sim 0.8^{\prime \prime}\right)$ from
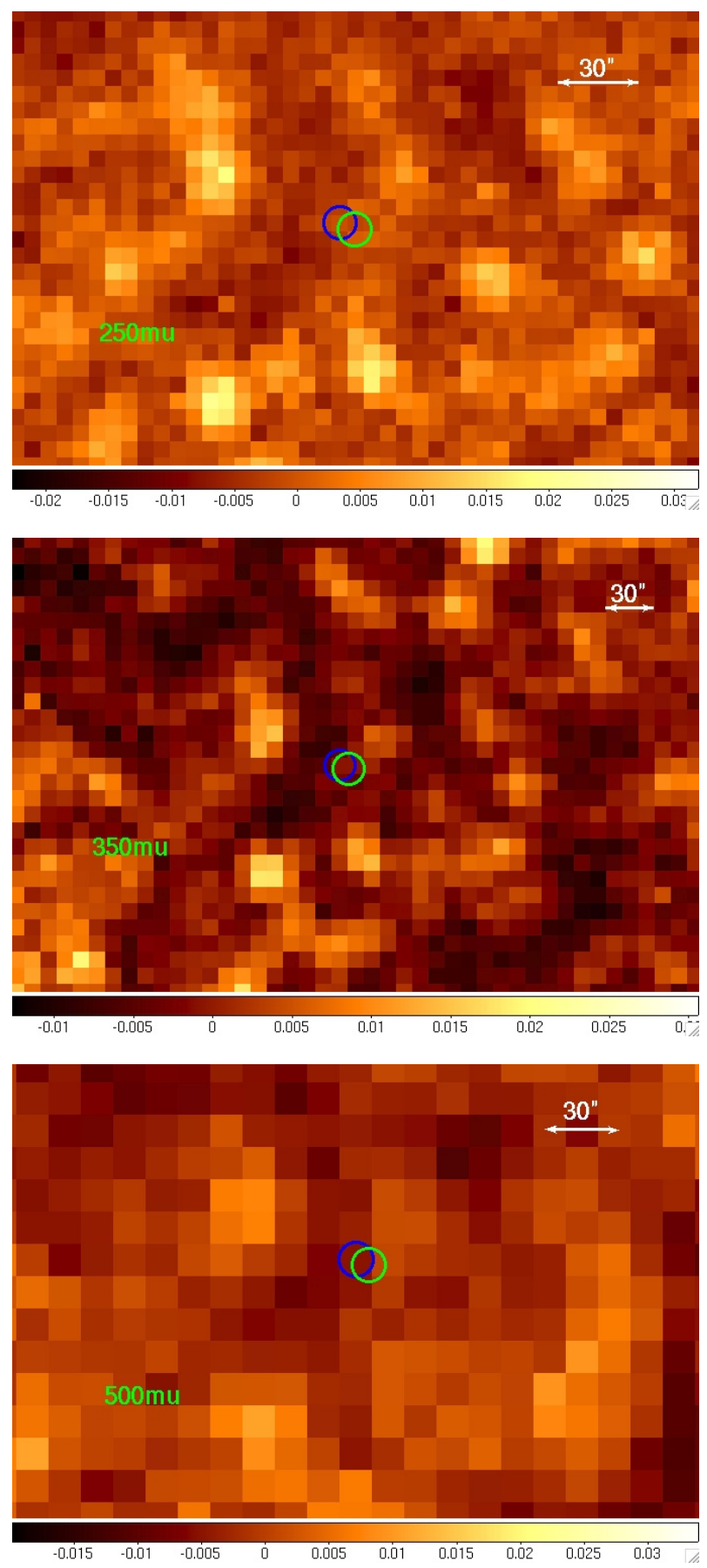

Fig. 1. The SPIRE images for SS1102. A blue circle marks the actual target position, while a green circle marks the nominal position. The image scale for $30^{\prime \prime}$ is shown at the top right. North is up, east is to the left.

the nominal position of 2M1207 (Fig. 2). This diffuse emission is at a flux level of $\sim 0.2 \mathrm{mJy}$, which is similar to that observed at the location of SS1102 in the $160 \mu \mathrm{m}$ data. We can consider this flux level to be the expected confusion noise in the $160 \mu \mathrm{m}$ band, based on which the detection of both 2M1207 and SS1102 at $160 \mu \mathrm{m}$ can be rejected.

\section{3. $2 M 1139$}

2M1139 is undetected in the PACS bands of 70 and $160 \mu \mathrm{m}$ (Fig. 3). The position uncertainty is $\sim 0.5$ pixel $\left(\sim 0.2^{\prime \prime}\right)$. We 

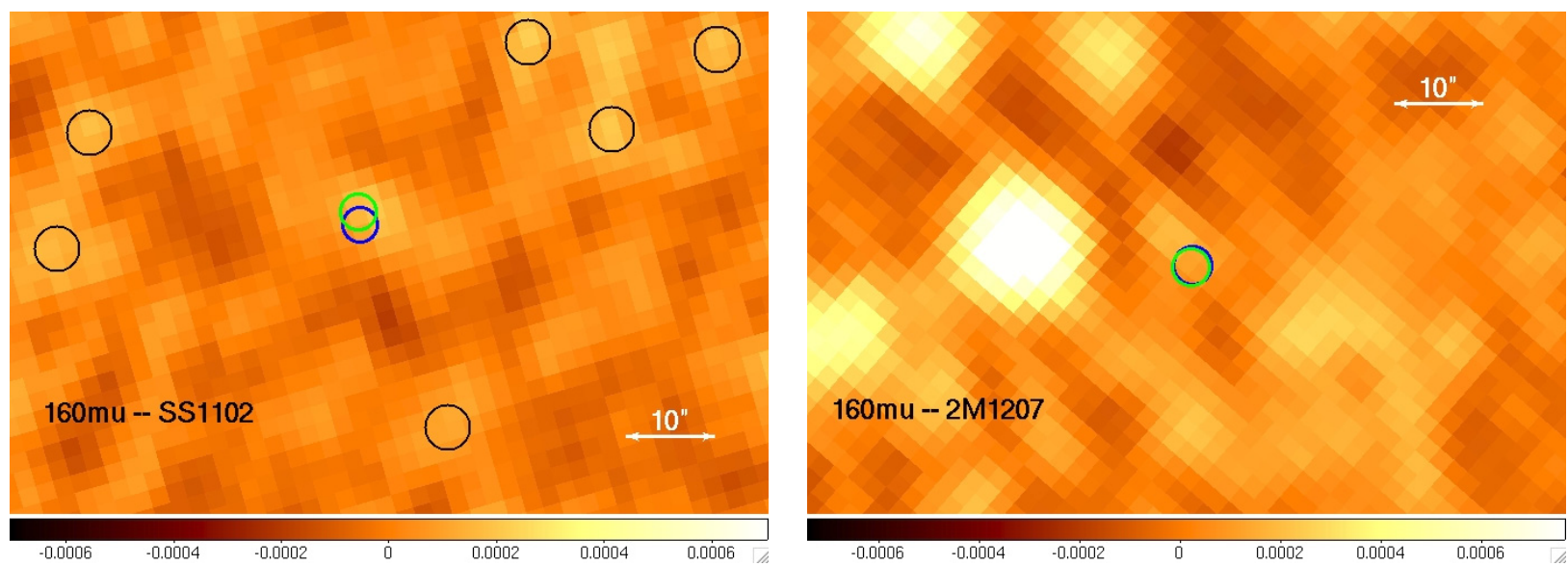

Fig. 2. The PACS $160 \mu \mathrm{m}$ images for SS1102 (top) and 2M1207 (bottom). A blue circle marks the actual target position, and a green circle marks the nominal position. For the SS1102 image, black circles indicate some of the confusion noise dominated regions with similar flux levels as observed at the nominal target position. The image scale for $10^{\prime \prime}$ is shown in the top right. North is up, east is to the left.
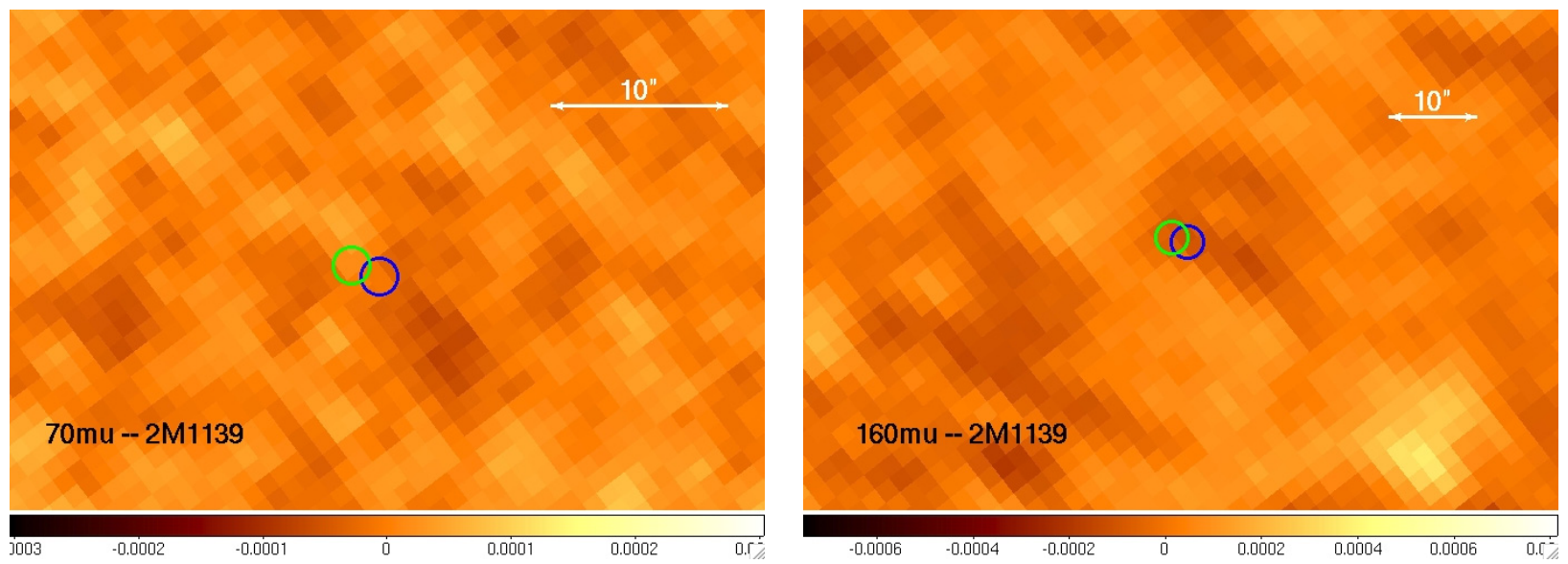

Fig. 3. PACS images for 2M1139. Blue circle marks the actual target position, green circle marks the nominal position. The image scale for $10^{\prime \prime}$ is shown at the top right. North is up, east is to the left.

Table 1. Observations.

\begin{tabular}{|c|c|c|c|}
\hline Band $^{a}$ & 2M1207 [Ref.] & $\mathrm{SS} 1102^{b}$ [Ref.] & 2M1139 [Ref.] \\
\hline$I$ & $1.13 \pm 0.1[1]$ & $0.83 \pm 0.1[1]$ & $1.17 \pm 0.1[1]$ \\
\hline$J$ & $10.10 \pm 0.89[2]$ & $9.75 \pm 1.0[2]$ & $13.43 \pm 0.3[2]$ \\
\hline$H$ & $11.35 \pm 1.0[2]$ & $11.7 \pm 1.0[2]$ & $16.3 \pm 0.3[2]$ \\
\hline$K$ & $11.12 \pm 0.98[2]$ & $11.72 \pm 1.0[2]$ & $16.7 \pm 0.3[2]$ \\
\hline $3.6 \mu \mathrm{m}$ & $8.49 \pm 0.32[3]$ & - & $12.08 \pm 0.7[3]$ \\
\hline $4.5 \mu \mathrm{m}$ & $7.15 \pm 0.26[3]$ & - & $8.5 \pm 0.6[3]$ \\
\hline $5.8 \mu \mathrm{m}$ & $6.36 \pm 0.06[3]$ & $7.6 \pm 0.2[6]$ & $5.98 \pm 0.6[3]$ \\
\hline $8 \mu \mathrm{m}$ & $5.74 \pm 0.21[3]$ & $6.74 \pm 0.2[6]$ & $3.88 \pm 0.5[3]$ \\
\hline $3.8 \mu \mathrm{m}$ & $7.00 \pm 0.6[5]$ & - & $10.23 \pm 1[5]$ \\
\hline $8.7 \mu \mathrm{m}$ & $5.60 \pm 1[6]$ & - & - \\
\hline $10.4 \mu \mathrm{m}$ & $7.50 \pm 1[6]$ & - & - \\
\hline $24 \mu \mathrm{m}$ & $4.32 \pm 0.03[3]$ & $3.96 \pm 0.1[3]$ & $1.10 \pm 0.2[3]$ \\
\hline $70 \mu \mathrm{m}$ & $9 \pm 4[4]$ & $9.4 \pm 0.6[7]$ & $<0.2[8]$ \\
\hline $160 \mu \mathrm{m}$ & $<0.2[8]$ & $<0.2[8]$ & $<0.075[8]$ \\
\hline $250 \mu \mathrm{m}$ & $<5.2[8]$ & $<1.5[8]$ & - \\
\hline $350 \mu \mathrm{m}$ & $<5[8]$ & $<1[8]$ & - \\
\hline $500 \mu \mathrm{m}$ & $<16[8]$ & $<1[8]$ & - \\
\hline
\end{tabular}

Notes. ${ }^{(a)}$ Fluxes in [mJy]. ${ }^{(b)}$ The fluxes at 5.8, 8, and $24 \mu \mathrm{m}$ are from the Spitzer/IRS spectrum.

References. 1 - DENIS; 2 - 2MASS; 3 - Riaz et al. (2006); 4 - Riaz \& Gizis (2008); 5 - Jayawardhana et al. (2003); 6 - Sterzik et. al. (2004); 7 - Harvey et al. (2012); 8 - this work. determined the upper limits by measuring the flux at the nominal position of the target. We note that the Spitzer $70 \mu \mathrm{m}$ upper limit reported in Riaz et al. (2009) is much higher than the PACS $70 \mu \mathrm{m}$ measurement, which can be expected given the higher confusion noise in the Spitzer MIPS bands compared to PACS.

\section{Disk modeling}

Figure 4 shows the model fits for SS1102 and 2M1207. The fits are based on a two-dimensional radiative transfer modeling of near-infrared to sub-millimeter data, using the code developed by Whitney et al. (2003). Detailed discussion of the fitting procedure and the variations in the model spectral energy distributions (SEDs) is provided in Riaz \& Gizis (2007). Here we provide a brief description of the best-fit models obtained, based on the lowest $\chi^{2}$ value. There are mainly six parameters related to the disk emission, which can be varied to obtain a good fit (Table 2 ). The disk scale-height varies as $h=h_{0}\left(\varpi / R_{*}\right)^{\beta}$, where $h_{0}$ is the scale-height at $R_{*}$ and $\beta$ is the flaring power. Both $2 \mathrm{M} 1207$ and SS1102 display flat disk structures, and values for $\beta$ of $\sim 1.1-1.2$, and $h_{0}$ of $\sim 0.3$ provide a good fit. We used the large grain model $\left(a_{\max }=1 \mathrm{~mm}\right)$ in the disk midplane and the medium-sized grains $\left(a_{\max } \sim 1 \mu \mathrm{m}\right)$ in the disk atmosphere. Since both disks display no silicate emission at $10 \mu \mathrm{m}$ (Riaz \& Gizis 2008), grains larger than the ISM are required to fit the flat silicate feature. 

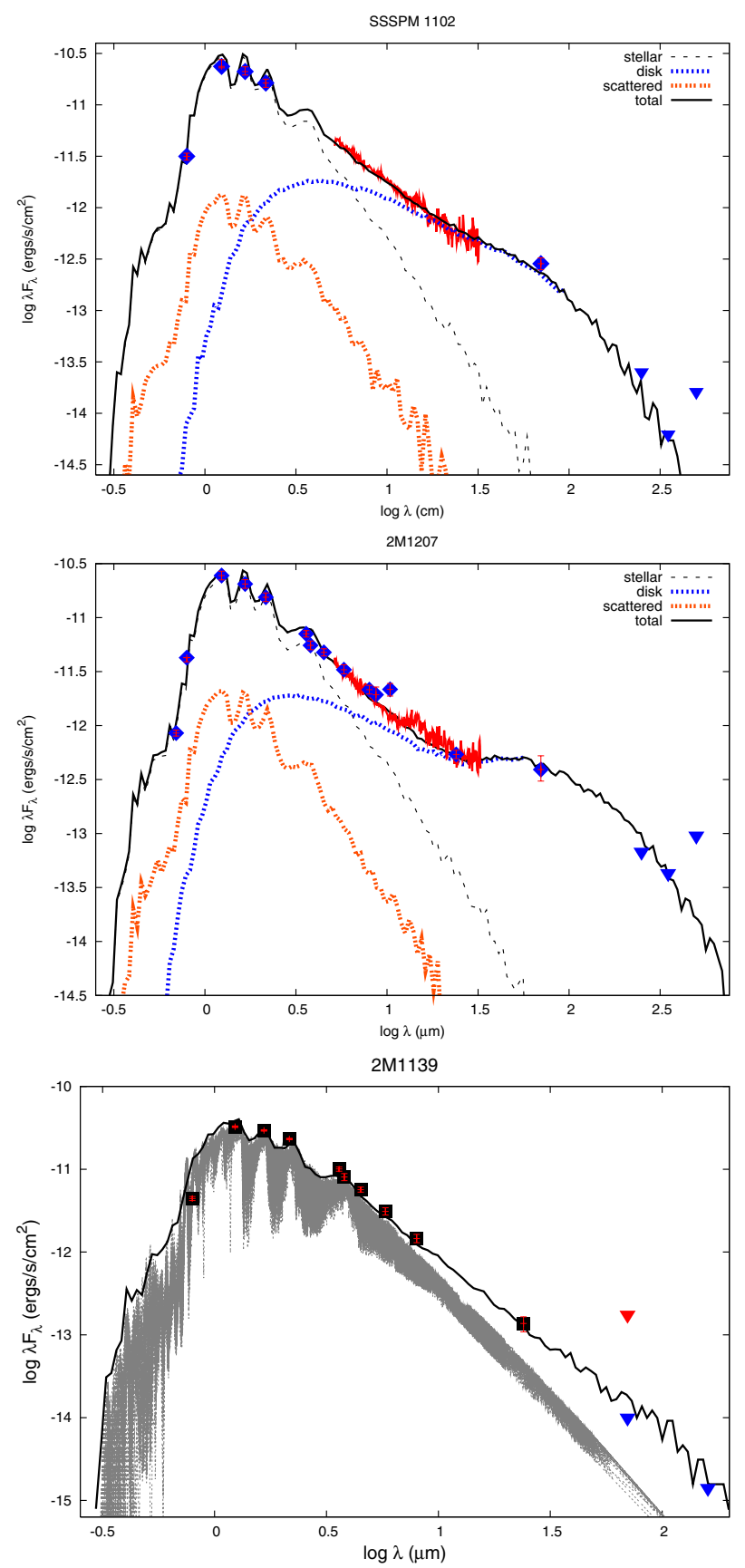

Fig. 4. The best-fit models for SS1102, 2M1207, and 2M1139 (bottom). Also shown is the contribution from the disk (blue) and the stellar photosphere (grey). The Spitzer/IRS spectrum is shown in red. Blue points are the Herschel upper limits. For 2M1139, the red point marks the Spitzer $70 \mu \mathrm{m}$ upper limit.

Further details of the variations in the model SEDs for the different grain models are discussed in Riaz \& Gizis (2007). Owing to the binning of photons in the models, there are a total of ten viewing angles available. For 2M1207, a good fit was obtained using close to edge-on inclination angles between $57^{\circ}$ and $69^{\circ}$, whereas SS1102 was modeled well using intermediate angles of $53^{\circ}-63^{\circ}$. We varied the inner disk radius $R_{\min }$ in multiples of $R_{\text {sub }}$, which is the dust sublimation radius. We obtained good fits for $R_{\text {min }}$ between 1 and $3 R_{\text {sub }}$, with a peak at $1 R_{\text {sub }}$ (based on a $\chi^{2}$ value comparison). This implies that there is an absence of an inner hole in the disk since it would have to be larger than the dust sublimation radius. Using the sub-mm upper limits, the best-fit model for both 2M1207 and SS1102 is that for an outer
Table 2. Disk parameters.

\begin{tabular}{lccc}
\hline \hline Parameter & $2 \mathrm{M} 1207$ & $\mathrm{SS} 1102$ & $2 \mathrm{M} 1139$ \\
\hline$\beta$ & $1.2 \pm 0.01$ & $1.12 \pm 0.01$ & $1.0 \pm 0.01$ \\
$h_{0}$ & $0.3 \pm 0.1$ & $0.25 \pm 0.1$ & $0.1 \pm 0.1$ \\
$R_{\min }$ & $1 R_{\text {sub }}\left(\sim 3 R_{*}\right)$ & $1 R_{\text {sub }}$ & $7 R_{\text {sub }}$ \\
$R_{\max }$ & $50-100 \mathrm{AU}$ & $50-100 \mathrm{AU}$ & $100 \mathrm{AU}$ \\
$M_{\text {disk }}$ & $<0.1 M_{\text {Jup }}$ & $<0.1 M_{\text {Jup }}$ & $<1 \mathrm{E}-7 M_{\text {Jup }}$ \\
$i$ & $57^{\circ}-69^{\circ}$ & $53^{\circ}-63^{\circ}$ & $50^{\circ}$ \\
\hline
\end{tabular}

disk radius, $R_{\max }$, of $50 \mathrm{AU}$ and an upper limit to the disk mass of $0.1 M_{\text {Jup }}$. A $100 \mathrm{AU}$ model for a mass of $0.5 M_{\text {Jup }}$ is also a good fit, though at a slightly higher $\chi^{2}$ value than the best fit. The 10 and 30 AU models are not a good fit to the longer part of the IRS spectrum for wavelengths $>10 \mu \mathrm{m}$. The optical depth is too large for these models even if the disk mass is reduced to $0.01 M_{\text {Jup }}$, and misses the $70-350 \mu$ m points. Increasing the disk mass for the same radius increases the optical depth beyond $\sim 30 \mu \mathrm{m}$, while increasing the radius for the same disk mass reduces the optical depth longward of $\sim 30 \mu \mathrm{m}$. The best fits for 2M1207 and SS1102 are the same as provided in Riaz \& Gizis (2008). We refer the readers to that paper for details on the rest of the fitting parameters.

We also attempted to model the $24 \mu \mathrm{m}$ excess along with the 70 and $160 \mu \mathrm{m}$ upper limits for 2M1139 (Fig. 4). We used the large grain model $\left(a_{\max }=1 \mathrm{~mm}\right)$ in both the disk midplane and the atmosphere to fit this source. The best fit is obtained for a disk mass of $1 \mathrm{E}-7 M_{\odot}$ and an outer disk radius of $100 \mathrm{AU}$. Since this is a transition disk with an inner opacity hole, a larger value of $R_{\min } \sim 7 R_{\text {sub }}$ provides a good fit. The parameter values listed in Table 2 for 2M1139 model fit should be considered with caution. These or any of the other disk parameters cannot be properly constrained, considering that we have only one excess point and two upper limits. This is particularly true in the case of the disk inclination angle and the outer disk radius. The model fit for 2M1139 mainly indicates that there may be a small mass of warm dust located at $\sim 1 \mathrm{AU}$ distance from the central brown dwarf.

\section{Discussion}

Figure 5 shows the extent of excess emission at $24 \mu \mathrm{m}$ for the TWA stars and brown dwarfs. The dot-dashed line represents the limit of $F_{24} / F_{K}$ under the assumption that both bands lie on the Rayleigh-Jeans tail of the stellar spectrum. The $F_{24}$-to- $F_{K}$ ratio for 2M1139 is higher than TWA 7 or TWA 13, indicating that there is a warmer debris disk around this brown dwarf than the cold $80 \mathrm{~K}$ disk around TWA 7, or the $65 \mathrm{~K}$ disk around TWA $13 \mathrm{AB}$. The dashed line represents a geometrically thin, optically thick flat disk with a spectral slope $\lambda F_{\lambda} \propto \lambda^{-4 / 3}$. Both 2M1207 and SS1102 lie almost precisely on the dashed line, indicating that there are optically thick flat disks around these two brown dwarfs. The primordial nature of the 2M1207 and SS1102 disks was also argued in Riaz et al. (2012b), based on the strength of the excess emission observed in the Spitzer IRAC bands and the IRS spectrum. That these disks are optically thick can also be inferred from the $K_{\mathrm{s}}$ to $24 \mu \mathrm{m}$ slope, $\alpha$, of the SED. We can consider a boundary of $\alpha=-2.2$ to separate the Class II and Class III sources (e.g., Luhman et al. 2010). 2M1207 and SS1102 have $\alpha$ of -1.38 and -1.42 , respectively, which is consistent with them being Class II systems, while $2 \mathrm{M} 1139$ has a $\alpha$ of -2.17 and is just above the Class III boundary. Large negative spectral-slope values can be expected for transition disks with photospheric emission shortward of $24 \mu \mathrm{m}$ owing to the 


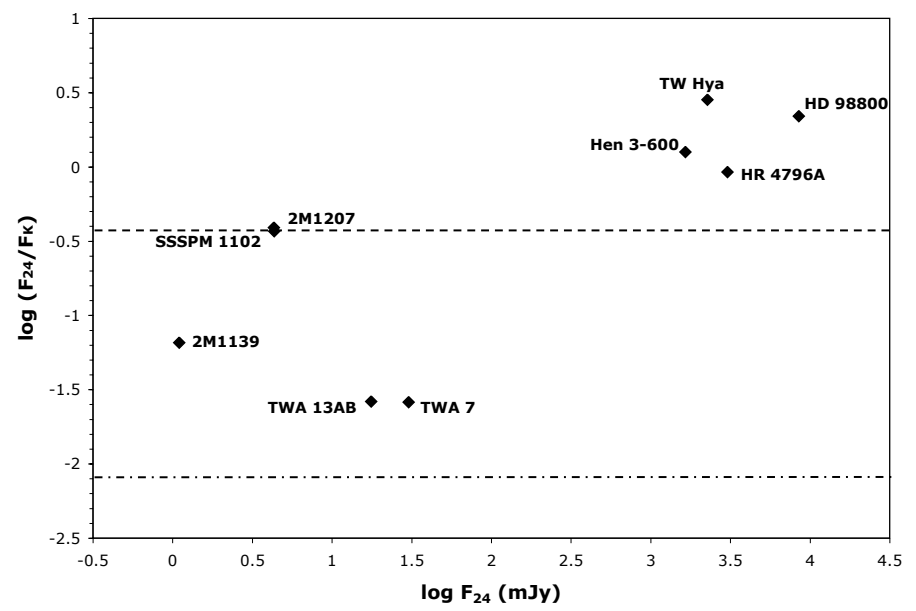

Fig. 5. The extent of excess emission at $24 \mu \mathrm{m}$ for the TWA stars and brown dwarfs. The dot-dashed line represents the limit of $F_{24} / F_{K}$ under the assumption that both bands lie on the Rayleigh-Jeans tail of the stellar spectrum. The dashed line represents a geometrically thin, optically thick flat disk with a spectral slope $\lambda F_{\lambda} \propto \lambda^{-4 / 3}$.

inner opacity holes (e.g., Cieza et al. 2010). Transition disks can be better characterized in a $\alpha_{\text {excess }}$ vs. $\lambda_{\text {turnoff }}$ diagram (Fig. 6), where $\lambda_{\text {turnoff }}$ is the longest wavelength at which the observed emission is photospheric, and $\alpha_{\text {excess }}$ is the spectral slope between $\lambda_{\text {turnoff }}$ and $24 \mu \mathrm{m}$ (e.g., Cieza et al. 2010). 2M1139 has a $\lambda_{\text {turnoff }}$ of $8 \mu \mathrm{m}$ and $\alpha_{\text {excess }}$ of -2.1 . The parameter $\lambda_{\text {turnoff }}$ is a good indicator of the size of the inner opacity hole in a transition disk. For a sub-stellar source, a $\lambda_{\text {turnoff }}$ of $8 \mu \mathrm{m}$ implies that there is an inner hole of $\sim 1 \mathrm{AU}$ (e.g., Muzerolle et al. 2006; Cieza et al. 2010). We have included in Fig. 6 the sample of young transition disks in the Ophichus and Taurus regions presented in Cieza et al. $(2010,2012)$. A large negative value for $\alpha_{\text {excess }}$ indicates that there is a debris disk or a continuous disk that has undergone significant grain growth and dust settling, in contrast to large positive values which correspond to an outer optically thick disk with a sharp inner cut due to e.g., the presence of a companion in the inner opacity hole. The location of 2M1139 in Fig. 6 is consistent with being a warm debris disk. It has a low fractional disk luminosity $\left(L_{\mathrm{D}} / L_{*}<10^{-4}\right)$ and is a non-accretor $\left(<10^{-11} M_{\odot} \mathrm{yr}^{-1}\right)$, similar to the debris disk candidates in the Ophichus and Taurus regions.

Figure 7 compares the mid-IR colors for the TWA brown dwarf disks with the Class II and the diskless sources in Taurus (from Luhman et al. 2010) and the transition disk samples in Ophichus and Taurus from Cieza et al. (2010, 2012). The fluxes at 3.6 and $4.5 \mu \mathrm{m}$ are not known for SS1102 and so it cannot be included in the top panel of Fig. 7. The dashed lines are boundaries used by Cieza et al. (2010) to separate the transition disks from the "full" disks with no inner holes. The colors for 2M1139 are similar to the sources classified as debris disks in these regions (black points), while 2M1207 and SS1102 lie among the Class II sources. Transition disks with flattened disk structures caused by grain growth/dust settling have redder [3.6]-[24] and [5.8]-[8] colors than the debris disks. While 2M1207 and SS1102 lie to the right of the transition disk boundary, their colors are also similar to disks dominated by grain growth/dust settling. This is consistent with the flat 10 and $20 \mu \mathrm{m}$ silicate emission features observed for both 2M1207 and SS1102 (Riaz \& Gizis 2008), which imply that there has been grain growth to sizes $>2 \mu \mathrm{m}$ followed by dust settling to the disk midplane. We can expect to measure a weaker excess in the [3.6]-[24] or [5.8]-[8] color for a $\sim 10 \mathrm{Myr}$ old disk than for the younger

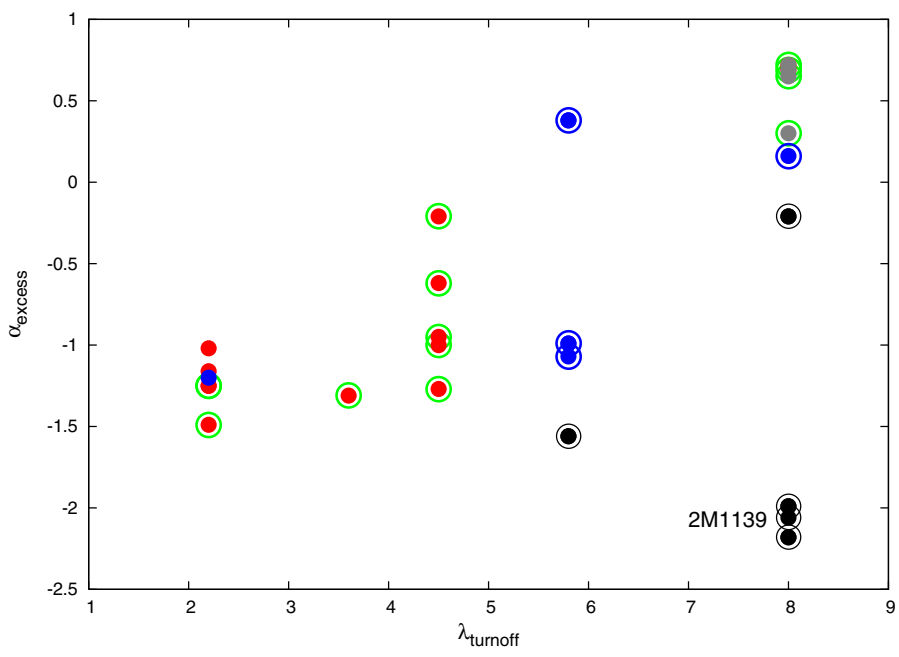

Fig. 6. A $\alpha_{\text {excess }}$ vs. $\lambda_{\text {turnoff }}$ diagram showing $2 \mathrm{M} 1139$ and the transition disk samples in Ophuichus and Taurus. Transition disks with inner holes due to grain growth/dust settling are indicated by red symbols, those due to photoevaporation by blue, and those due to giant planet formation by grey. Debris disks are indicated by black filled symbols. Open green circles indicate objects with accretion rates of $1 \mathrm{E}-11$ to $1 \mathrm{E}-9 M_{\odot} \mathrm{yr}^{-1}$ and values of $L_{\mathrm{D}} / L_{*}$ between $1 \mathrm{E}-3$ to $1 \mathrm{E}-2$. Blue open circles indicate non-accretors $\left(<1 \mathrm{E}-11 M_{\odot} \mathrm{yr}^{-1}\right)$ with $L_{\mathrm{D}} / L_{*}$ between $1 \mathrm{E}-3$ and $1 \mathrm{E}-2$. Black open circles indicate non-accretors $\left(<1 \mathrm{E}-11 M_{\odot} \mathrm{yr}^{-1}\right)$ with weak disks $\left(L_{\mathrm{D}} / L_{*}<1 \mathrm{E}-3\right)$.

1 Myr Class II sources in Taurus, most of which are still dominated by sub-micron sized dust grains (Riaz 2009; Sargent et al. 2009).

There are four widely discussed mechanisms to explain the formation of inner opacity holes in transition disks. The process of giant planet formation would require a high disk mass ( $>1 M_{\text {Jup }}$ ) and therefore can be rejected for 2M1139. Tidal truncation due to a close companion may also not be applicable for 2M1139, as Chauvin et al. (2010) have found no secondaries for this object at projected separations of 5-240 AU. However, given an inner hole size of $\sim 1 \mathrm{AU}$ for $2 \mathrm{M} 1139$, we cannot rule out the possibility of a tight binary surrounded by a circumbinary debris disk. We note that there is no report that $2 \mathrm{M} 1139$ is a close double in the limited set of published high-resolution spectra. Inner disk evacuation caused by significant grain growth and dust settling is expected to exacerbate accretion, resulting in an actively accreting disk with an inner opacity hole (e.g., Cieza et al. 2010). This mechanism would also not be applicable for $2 \mathrm{M} 1139$. The transition from a primordial to a debris stage could take place due to the photoevaporation mechanism, which can set in once the accretion rate drops below the photoevaporative wind rate. As soon as photoevaporation sets in, the remaining gas in the disk is removed very rapidly ( $\ll 1 \mathrm{Myr})$, and what remains is a gas-poor debris disk (e.g., Alexander \& Armitage 2006). For disks that are still in the process of photoevaporation, we can expect weak or negligible accretion but higher fractional disk luminosities than $2 \mathrm{M} 1139$, that are on the order of $10^{-2}$ (Fig. 6). The much lower $L_{\mathrm{D}} / L_{*}$ for $2 \mathrm{M} 1139$ thus suggests that it has already made a transition to the debris phase. Interestingly, while the $24 \mu \mathrm{m}$ excess for $2 \mathrm{M} 1139$ is larger than that observed for the debris disks TWA 7 and 13 (Fig. 5), it remains undetected at $70 \mu \mathrm{m}$ and beyond. This is unlike TWA 7 and 13, which have strong excesses at $70 \mu \mathrm{m}$ (Low et al. 2005). This suggests that there is an absence of cold dust material in 2M1139, and perhaps that the outer disk region has been completely dissipated. We cannot, however, conclude that there is no cold dust material 

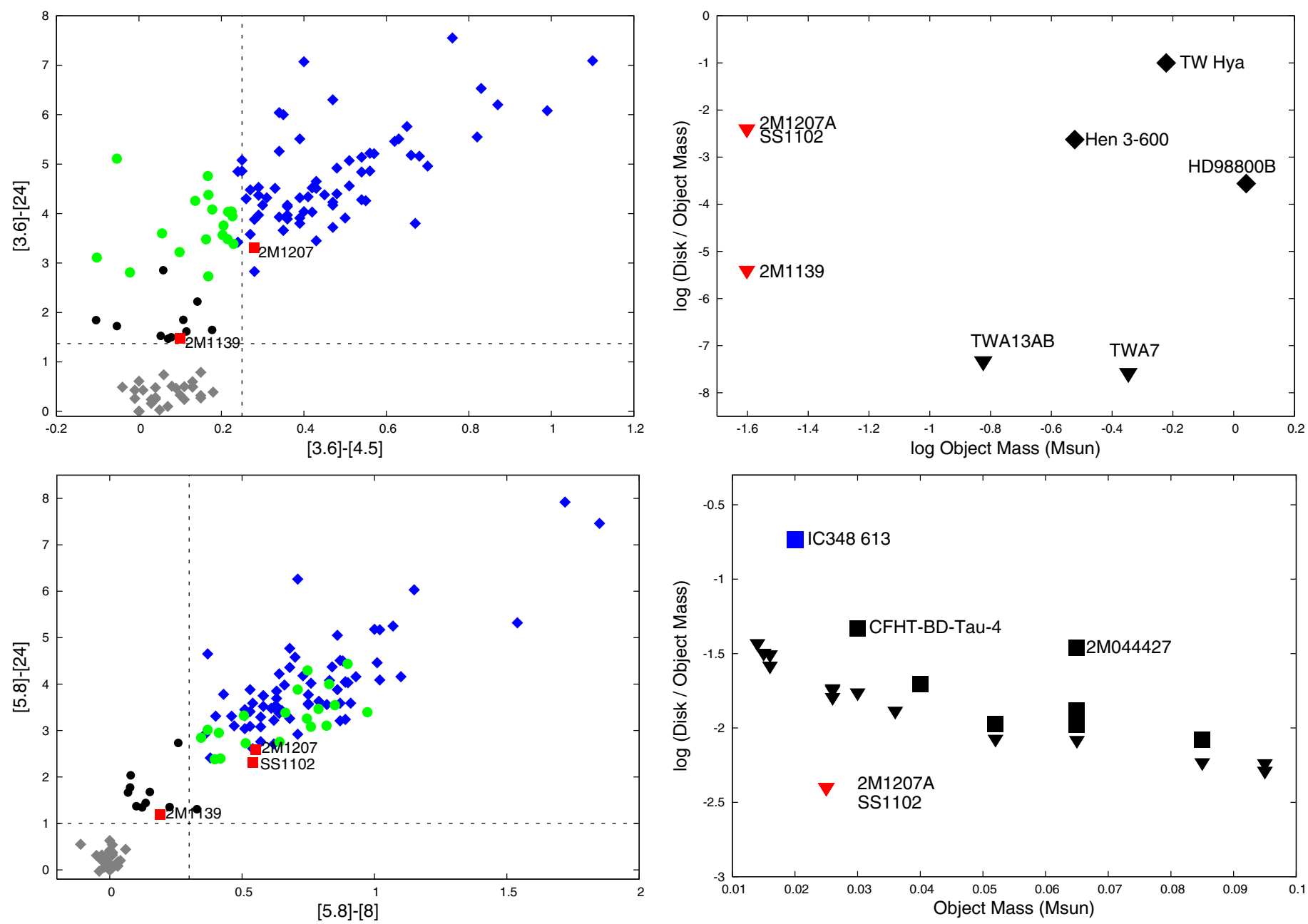

Fig. 7. Mid-IR color-color diagram for TWA brown dwarf disks. Also included for comparison are the diskless (grey) and Class II (blue) sources in Taurus, and the transition disk sample in Ophuichus and Taurus. Green symbols indicate the transition disks with inner disk evacuation due to grain growth/dust settling. Black symbols indicate the debris disks.

surrounding 2M1139, or that this is a case of inner disk clearing due to photoevaporation. There is a limit to the fractional disk luminosity that can be reached with a certain exposure time. We estimate that we would be able to detect an AU Mic like disk $\left(L_{\mathrm{D}} / L_{*} \sim 6 \mathrm{E}-4\right.$; Liu et al. 2004$)$ with the $\sim 1 \mathrm{~h}$ total exposure time that we used for $2 \mathrm{M} 1139$. The fractional disk luminosity as estimated from the 2M1139 SED is of the order of $\sim 10^{-5}$, which is well below the detection limit that can be obtained with our present setup.

Some results have suggested two distinct populations in the TWA, corresponding to a bimodal distribution in the rotation periods (Lawson \& Crause 2005) and the presence of warm $(T \gtrsim 100 \mathrm{~K})$ dust (Low et al. 2005; Weinberger et al. 2004). Low et al. found negligible amounts of warm dust around 20 of their 24 TWA targets, while the other 4 display strong excess emission at $24 \mu \mathrm{m}$ (Fig. 5). Lawson \& Crause measured the projected rotational velocities, $v \sin i$, for the TWA 1-13 group led by TW Hya to be $3-10 \mathrm{~km} \mathrm{~s}^{-1}$, while the velocities for the stars in the TWA 14-19 group are $\sim 20-50 \mathrm{~km} \mathrm{~s}^{-1}$. These authors suggested that the former group may be younger than the latter by $\sim 8 \mathrm{Myr}(8-10 \mathrm{Myr}$ versus $\sim 17 \mathrm{Myr})$. 2M1139 has a $v \sin i$ of $25 \mathrm{~km} \mathrm{~s}^{-1}$, which is a factor of $\sim 2$ higher than $2 \mathrm{M} 1207$ (13 $\mathrm{km} \mathrm{s}^{-1}$; Scholz et al. 2005). This brown dwarf thus could be

Fig. 8. Top - a) relative disk masses vs. object mass for TWA disks. Bottom - b) a comparison of TWA relative disk masses with other known brown dwarf disks in Taurus and IC 348. Upper limits are shown as bold triangles.

slightly older than 2M1207, which could explain its advanced evolutionary stage of being a debris disk, compared to the primordial colors observed for 2M1207 and SS1102. We note that a variety of SED morphologies is observed at any given age (e.g., Hartmann et al. 2005), and the presence of a debris disk does not necessarily represent an older age.

$2 \mathrm{M} 1207$ is known to have a $\sim 5 M_{\text {Jup }}$ secondary that lies at a projected separation of $\sim 55 \mathrm{AU}$ (Chauvin et al. 2004). The best model fit for 2M1207 is obtained for an outer disk radius of $50 \mathrm{AU}$, which suggests that there has been tidal truncation of the disk by the massive companion. This truncation could be responsible for the sub-mm non-detection of this disk. However, we cannot rule out that the non-detection for both 2M1207 and SS1102 in the SPIRE bands is caused by the limitations of the confusion noise. We discussed in Riaz et al. (2012a) the possibility of the formation of the $\sim 5 M_{\text {Jup }}$ secondary in the $2 \mathrm{M} 1207$ disk via disk fragmentation. Our argument is that even a very low mass disk could produce a fragment of $\sim 0.035 M_{\text {Jup }}$, which can then grow over time to form a $5 M_{\text {Jup }}$ mass object. The main requirement for such a case is for the initial mass of the disk to be higher than its current estimate (at least 10-20 $M_{\text {Jup }}$ ). The upper limit to the disk mass of $\sim 0.1 M_{\text {Jup }}$ obtained from modeling the 2M1207 SED thus does not rule out disk fragmentation, since the system is relatively old and we have not observed it during its early stages when fragmentation might have occurred 
$(<0.1 \mathrm{Myr})$. This system may be considered as a very low-mass wide binary rather than a planetary system, and some alternative formation mechanisms, such as the turbulent fragmentation of a core, were discussed in Riaz et al. (2012a).

Figure 8a compares the relative disk mass upper limits for 2M1207, SS1102, and 2M1139 with the other disk sources in the TWA (using data from Calvet et al. 2002; Low et al. 2005; Andrews et al. 2010). TW Hya is known to be a strong accretor, with $\dot{M}$ of $4 \times 10^{-10} M_{\odot} \mathrm{yr}^{-1}$ (Muzerolle et al. 2000). Hen 3-600 is a weaker accreting source $\left(0.5 \times 10^{-10} M_{\odot} \mathrm{yr}^{-1}\right)$, while the other disk sources in TWA are non-accretors (e.g., Muzerolle et al. 2000). Among the brown dwarfs, 2M1207 has a variable accretion activity of $0.8-1.6 \times 10^{-10} M_{\odot} \mathrm{yr}^{-1}$ (Scholz et al. 2005; Stelzer et al. 2007), while SS1102 and 2M1139 are non-accretors $\left(<10^{-11} M_{\odot} \mathrm{yr}^{-1}\right.$; Scholz et al. 2005). The disk mass limits for 2M1207 and SS1102 are comparable to the weak/non-accretors in the TWA, while 2M1139 is more similar to the other two transition disks TWA 7 and 13. Transition disks are found to have much lower disk masses and accretion rates than non-transition disk sources (e.g., Cieza et al. 2010). Both accreting/non-accreting objects, or sources with or without mid-IR excess are found to have an equal likelihood of a sub-mm detection (e.g., Lee et al. 2011). The lack of detection in the SPIRE bands for 2M1207 and SS1102 could be due to a paucity of millimeter sized dust grains in these disks. Figure $8 \mathrm{~b}$ compares the relative disk masses for the Taurus, IC 348, and TWA brown dwarfs, which thus cover an age range of 1-10 Myr. The Taurus and IC 348 disk mass measurements are taken from Scholz et al. (2006) and Klein et al. (2003), respectively. The TWA disks have relative masses smaller than nearly all brown dwarf disks in Taurus, thus suggesting that the disk mass may depend on the age of the system. For higher mass stars $\left(\sim 0.1-1 M_{\odot}\right)$ in the Taurus, Ophiuchus, and IC 348 clusters, the disk mass distribution for the relatively older 2-3 Myr clusters is found to be shifted by a factor of $\sim 20$ to lower masses, compared to the distribution for the $\sim 1$ Myr old clusters (Lee et al. 2011). For the case of the brown dwarfs, most of the data points in Fig. 8b for object masses $<0.03 M_{\odot}$ are upper limits. The non-detection of these faint objects in the sub- $\mathrm{mm} / \mathrm{mm}$ bands could be due to the low sensitivity of the observations. To probe any dependence of the disk mass on age would require an analysis of larger samples of brown dwarfs across a wider range in ages.

\section{Summary}

We have reported the non-detection of the TWA brown dwarf disks SS1102 and 2M1207 in the SPIRE and PACS $160 \mu \mathrm{m}$ bands, and a non-detection of 2M1139 in the PACS 70 and $160 \mu \mathrm{m}$ bands. We have proposed that 2M1139 likely possesses a warm debris disk, as indicated by its $24 \mu \mathrm{m}$ excess emission. We have estimated an upper limit to the disk mass for SS1102 and 2M1207 of $0.1 M_{\text {Jup }}$, which places them among the weaker brown dwarf disks in the $\sim 1$ Myr old Taurus region, and suggests that there is a decline in the brown dwarf disk mass with the age of the system.

\section{References}

Alexander, R., \& Armitage, P. 2006, ApJ, 639, L83

Andrews, S., Czekala, I., Wilner, D., et al. 2010, ApJ, 710, 462

Barrado y Navascués, D. 2006, A\&A, 459, 511

Calvet, N., D’Alessio, P., Hartmann, L., et al. 2002, ApJ, 568, 1008

Chauvin, G., Lagrange, A.-M., Dumas, C., et al. 2004, A\&A, 425, L29

Chauvin, G., Lagrange, A.-M., Bonavita, M., et al. 2010, A\&A, 509, A52

Cieza, L. A., Schreiber, M. R., Romero, G. A., et al. 2010, ApJ, 712, 925

Cieza, L. A., Schreiber, M. R., Romero, \& Gisela A. 2012, ApJ, 750, 157

Gizis, J., Jao, W., Subasavage, J., \& Henry, T. 2007, ApJ, 669, 45

Griffin, M. J., Abergel, A., Abreu, A., et al. 2010, A\&A, 518, L3

Hartmann, L., Megeath, S. T., Allen, L., et al. 2005, ApJ, 629, 881

Harvey, P., Henning, T., Ménard, F., et al. 2012, ApJ, 744, L1

Jayawardhana, R., Hartmann, L., Fazio, G., et al. 1999, ApJ, 521, L129

Jayawardhana, R., Ardila, D. R., Stelzer, B., \& Haisch, K. E., Jr. 2003, AJ, 126, 1515

Kastner, J. H., Zuckerman, B., Weintraub, D. A., \& Forveille, T. 1997, Science, 277, 67

Klein, R., Apai, D., Pascucci, I., Henning, Th., \& Waters, L. B. F. M. 2003, ApJ, 593, L57

Lawson, W. A., \& Crause, L. A., 2005, MNRAS, 357, 1399

Lee, N., Williams, J. P., \& Cieza, L. A. 2011, ApJ, 736, 135

Liu, M. C., Matthews, B. C., Williams, J. P., \& Kalas, P. G. 2004, ApJ, 608, 526 Low, F. J., Smith, P. S., Werner, M., et al. 2005, ApJ, 631, 1170

Luhman, K., Allen, P. R., Espaillat, C., Hartmann, L., \& Calvet, N. 2010, ApJS, 186,111

Muzerolle, J., Calvet, N., Briceo, C., Hartmann, L., \& Hillenbrand, L. 2000, ApJ, 535, L47

Muzerolle, J., Adame, L., D’Alessio, P., et al. 2006, ApJ, 643, 1003

Pilbratt, G., Riedinger, J. R., Passvogel, T., et al. 2010, A\&A, 518, L1

Poglitsch, A., Waelkens, C., Geis, N., et al. 2010, A\&A, 518, L2

Riaz, B., \& Gizis, J. 2008, ApJ, 681, 1584

Riaz, B., Gizis, J., \& Hmiel, A. 2006, ApJ, 639, L79

Riaz, B., Lodieu, N., \& Gizis, J. 2009, ApJ, 705, 1173

Riaz, B., Lodato, G., Stamatellos, D., \& Gizis, J. E. 2012a, MNRAS, 422, L6; Erratum: 2012, MNRAS, 424, 74

Riaz, B., Lodieu, N., Goodwin, S., Stamatellos, D., \& Thompson, M. 2012b, MNRAS, 420, 2497

Scholz, A., Jayawardhana, R., \& Brandeker, A. 2005, ApJ, 629, L41

Scholz, A., Jayawardhana, R., \& Wood, K. 2006, ApJ, 645, 1498

Stelzer, B., Scholz, A., \& Jayawardhana, R. 2007, ApJ, 671, 842

Sterzik, M. F., Pascucci, I., Apai, D., van der Bliek, N., \& Dullemond, C. P. 2004, A\&A, 427, 245

Weinberger, A. J., Becklin, E. E., Zuckerman, B., \& Song, I. 2004, AJ, 127, 2246

Whitney, B., Wood, K., Bjorkman, J., \& Cohen, M. 2003, ApJ, 598, 1079 